\title{
JENDL-4.0/HE Benchmark Test with Concrete and Iron Shielding Experiments at JAEA/TIARA
}

\author{
Chikara Konno ${ }^{1 *}$, Norihiro Matsuda ${ }^{1}$, Saerom Kwon ${ }^{2}$, Masayuki Ohta $^{2}$, and Satoshi Sato ${ }^{2}$ \\ ${ }^{1}$ Japan Atomic Energy Agency, Nuclear Science and Engineering Center, 319-1195 Tokai-mura, Japan \\ ${ }^{2}$ National Institutes for Quantum and Radiological Science and Technology, Fusion Energy Research and Development Diretorate, \\ 039-3212 Rokkasho-mura, Japan
}

\begin{abstract}
As a benchmark test of JENDL-4.0/HE released in 2015, we have analyzed the concrete and iron shielding experiments with the quasi mono-energetic 40 and $65 \mathrm{MeV}$ neutron sources at TIARA in JAEA by using MCNP5 and ACE files processed from JENDL-4.0/HE with NJOY2012. As a result, it was found out that the calculation results with JENDL-4.0/HE agreed with the measured ones in the concrete experiment well, while they underestimated the measured ones in the iron experiment with $65 \mathrm{MeV}$ neutrons more for the thicker assemblies. We examined the ${ }^{56} \mathrm{Fe}$ data of JENDL-4.0/HE in detail and it was considered that the larger non-elastic scattering cross sections of ${ }^{56} \mathrm{Fe}$ caused the underestimation in the calculation with JENDL-4.0/HE for the iron experiment with $65 \mathrm{MeV}$ neutrons.
\end{abstract}

\section{Introduction}

A Japanese Evaluated Nuclear Data Library (JENDL) special purpose file, JENDL-4.0 High Energy File (JENDL-4.0/HE) [1], is an extended version of JENDL4.0 for incident neutrons up to $200 \mathrm{MeV}$ for 130 nuclei, and a nuclear data library for incident protons up to 200 $\mathrm{MeV}$ for 133 nuclei, which are relevant to high-energy accelerator applications. It was released in November, 2015.

More than 20 years ago shielding experiments at the Takasaki Ion Accelerators for Advanced Radiation Application (TIARA) in Japan Atomic Energy Agency (JAEA) were carried out for concrete and iron with quasimono energetic 40 or $65 \mathrm{MeV}$ neutrons produced by bombarding 43 or $68 \mathrm{MeV}$ protons to a ${ }^{7} \mathrm{Li}$ target, which were collimated and injected to a test shield of $1.2 \mathrm{~m} \mathrm{x} 1.2$ $\mathrm{m}$ as shown in Fig. 1 [2]. The neutron spectra above 10 $\mathrm{MeV}$ just behind the test concrete or iron shield on beam axis were measured with a BC501A scintillation detector. These experiments are very useful for benchmarking higher energy neuron incident nuclear data libraries [3]. So far we benchmarked FENDL-3.0 [4], JENDL/HE2007 [5] and ENDF/B-VII.1 [6] with these experiments and deduced the following conclusions [7].

1) Concrete experiments : The calculation result with JENDL/HE-2007 agrees with the measured one best. Those with FENDL-3.0 and ENDF/B-VII.1 drastically overestimate the measured data. It is confirmed that this overestimation is due to both the larger elastic and smaller non-elastic scattering data of ${ }^{16} \mathrm{O}$ in FENDL3.0 and ENDF/B-VII.1.

2) Iron experiments : The calculation result with FENDL3.0 agrees with the measured one best. That with

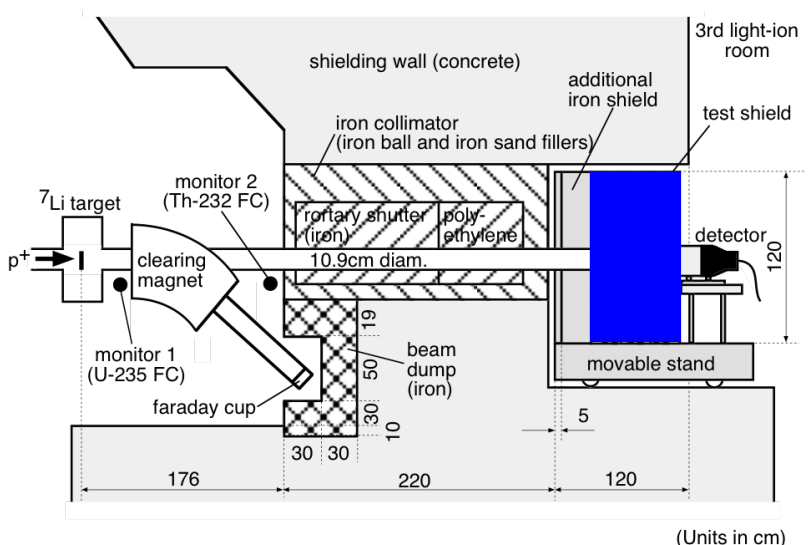

Fig. 1. Experimental configuration of TIARA shielding experiments.

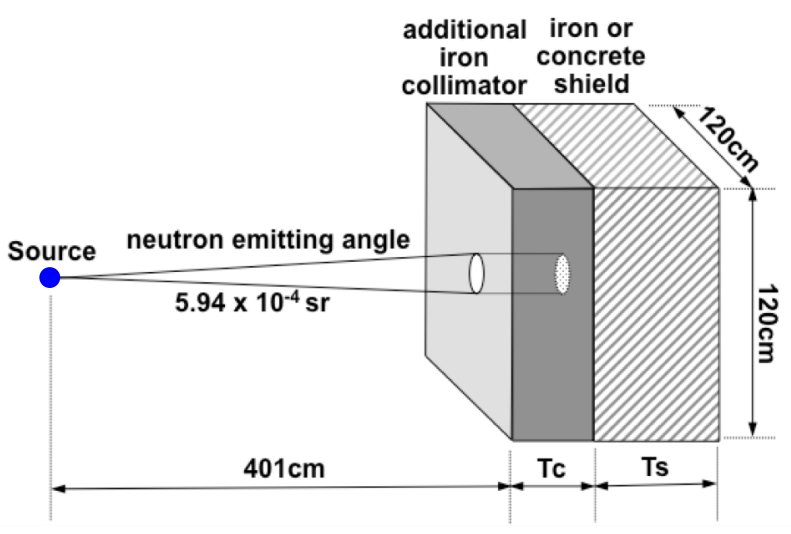

Fig. 2. Calculation model.

\footnotetext{
Corresponding author: konno.chikara@jaea.go.jp
} 
JENDL/HE-2007 fairly agrees with the experimental data. That with ENDF/B-VII.1 drastically overestimates the measured one, which is confirmed to be due to the smaller non-elastic scattering data of ${ }^{56} \mathrm{Fe}$ in ENDF/B-VII.1.

In this study we carry out a benchmark test of JENDL4.0/HE with the concrete and iron shielding experiments at TIARA in JAEA.

\section{Analysis}

The measured spectrum data of quasi-mono energetic 40 or $65 \mathrm{MeV}$ neutrons were adopted as the source neutron in the analysis. Only the collimated neutron beam and test shield (iron or concrete) with additional shields were modeled in the analysis as shown in Fig. 2. The measured neutron spectra above $10 \mathrm{MeV}$ just behind the test shield on beam axis were compared with the calculated ones in this study.

The Monte Carlo code MCNP-5.14 [8] and the ACE file processed from JENDL-4.0/HE with the NJOY2012.50 [9] code by ourselves were used in the analysis. We also used the official ACE files of JENDL/HE-2007 [10] and FENDL-3.1b (the revised FENDL-3.0) [11]. FENDL-3.1b was used only for the iron experiment, because it was pointed out that ${ }^{16} \mathrm{O}$ in FENDL-3.0 had some problems [7] and the ${ }^{16} \mathrm{O}$ was not revised in FENDL-3.1b. ENDF/B-VII.1 was not used because of defects of the ${ }^{56} \mathrm{Fe}$ and ${ }^{16} \mathrm{O}$ data [7].

\section{Results and discussion}

\subsection{Concrete experiment with $40 \mathrm{MeV}$ neutrons}

Figure 3 shows the measured and calculated neutron spectra in the concrete experiment with $40 \mathrm{MeV}$ neutrons. The agreement between the measured and calculated spectra is roughly good. For more quantitative comparison of the overall tendency, Figs. 4 and 5 show the ratios $(\mathrm{C} / \mathrm{Es})$ of the calculated peak $(35-45 \mathrm{MeV})$ and continuum (10 - $35 \mathrm{MeV})$ neutron fluxes to the measured ones, respectively, where "Expt. error" means an experimental error estimated from the errors of the measured neutron spectra by assuming no correlation among the measured flux errors in Fig. 3 because no correlation data among the measured flux errors were not specified in Ref. 2. The calculation results with JENDL/HE-2007 and JENDL-4.0/HE agree with the measured one well, though the calculation result with JENDL-4.0/HE is slightly better than that with JENDL/HE-2007.

\subsection{Concrete experiment with $65 \mathrm{MeV}$ neutrons}

The measured and calculated neutron spectra in the concrete experiment with $65 \mathrm{MeV}$ neutrons are shown in Fig. 6. The calculated spectra generally represent the measured ones. Figures 7 and 8 show more quantitative

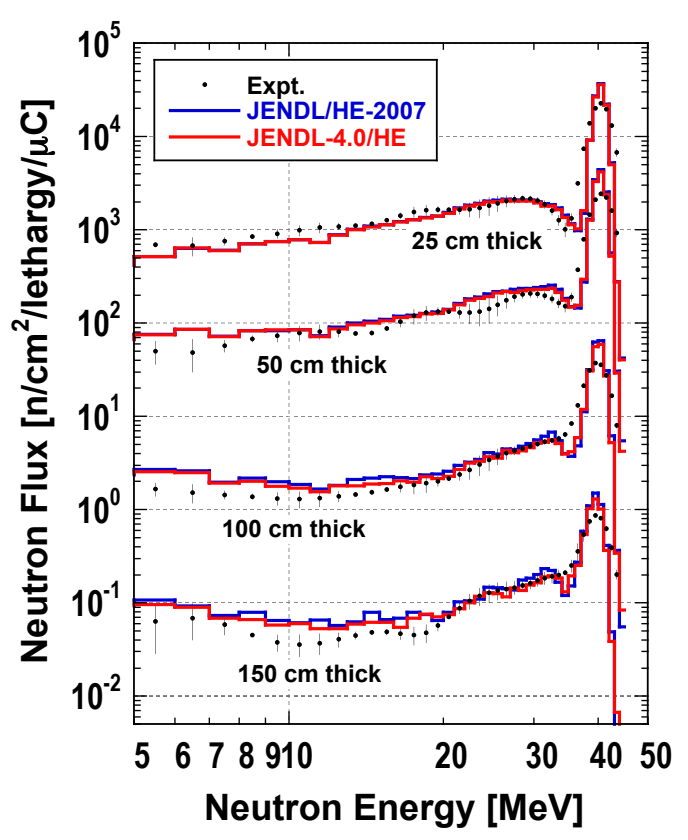

Fig. 3. Measured and calculated neutron spectra in concrete experiment with $40 \mathrm{MeV}$ neutrons.

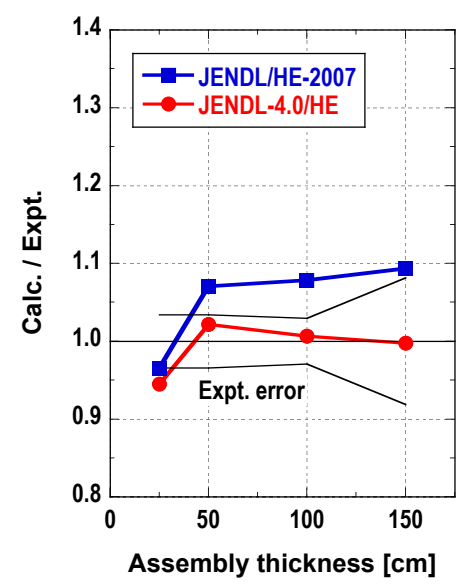

Fig. 4. C/Es of peak (35-45 MeV) neutron fluxes in concrete experiment with $40 \mathrm{MeV}$ neutrons.

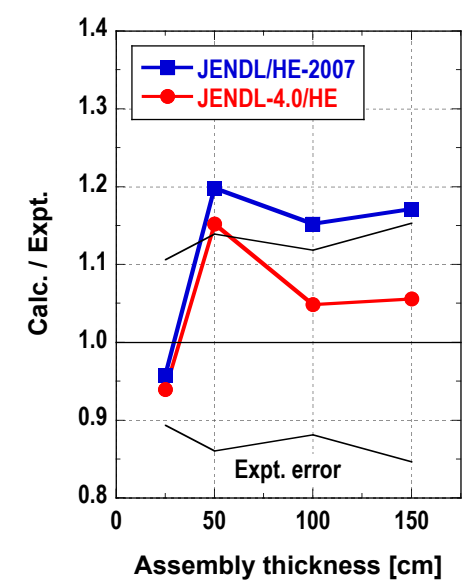

Fig. 5. C/Es of continuum (10-35 MeV) neutron fluxes in concrete experiment with $40 \mathrm{MeV}$ neutrons. 


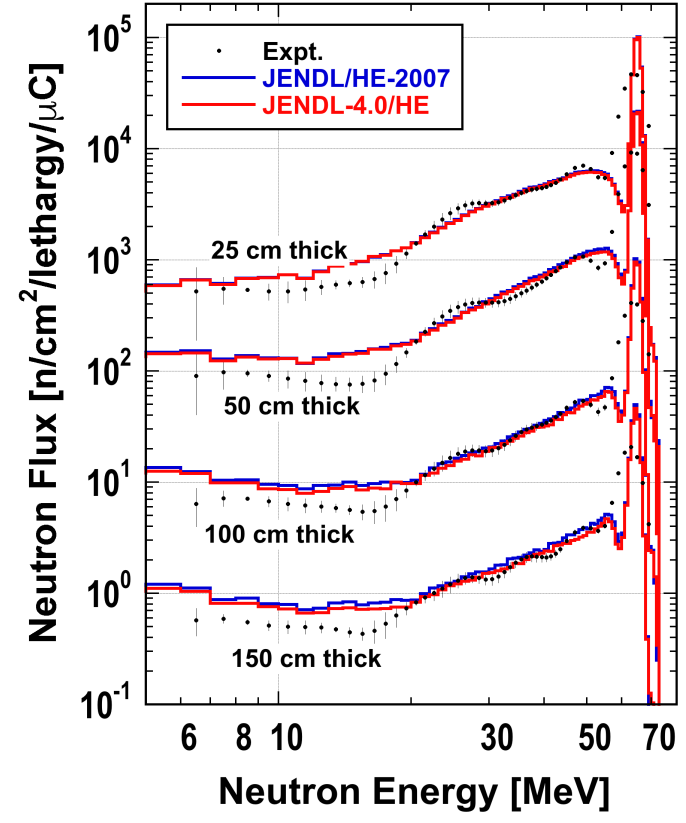

Fig. 6. Measured and calculated neutron spectra in concrete experiment with $65 \mathrm{MeV}$ neutrons.

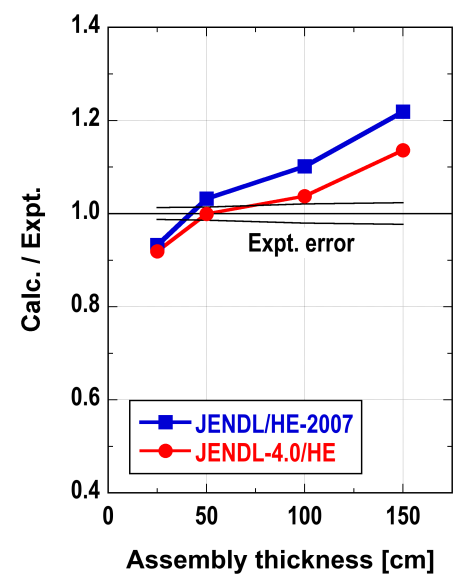

Fig. 7. C/Es of peak (60-70 MeV) neutron fluxes in concrete experiment with $65 \mathrm{MeV}$ neutrons.

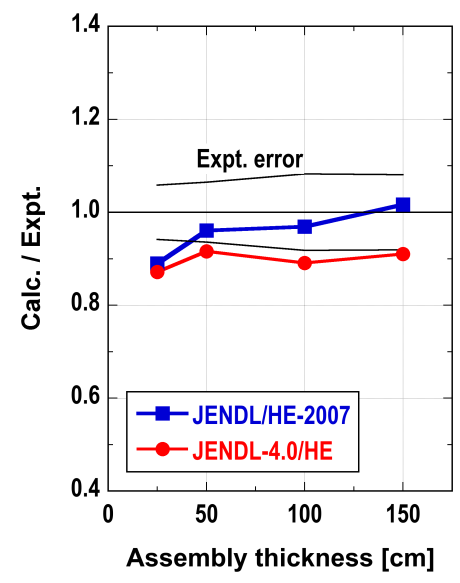

Fig. 8. $\mathrm{C} /$ Es of continuum (10-60 MeV) neutron fluxes in concrete experiment with $65 \mathrm{MeV}$ neutrons. comparison between the measured and calculated neutron fluxes, i.e., the $\mathrm{C} / \mathrm{Es}$ of the peak $(60-70 \mathrm{MeV})$ and continuum (10 - $60 \mathrm{MeV})$ neutron fluxes, respectively. The calculation results with JENDL/HE-2007 and JENDL-4.0/HE agree with the measured one well.

It is concluded that the JENDL-4.0/HE data are good for concrete.

\subsection{Iron experiment with $40 \mathrm{MeV}$ neutrons}

Figure 9 shows the measured and calculated neutron spectra in the iron experiment with $40 \mathrm{MeV}$ neutrons. The calculated neutron spectra with FENDL-3.1b, JENDL/HE-2007 and JENDL-4.0/HE agree with the measured one well, though a small strange step, which is probably caused by discontinuity between data below and above $20 \mathrm{MeV}$, appears around $20 \mathrm{MeV}$ in that in FENDL-3.1b. The quantitative comparison between the measured and calculated neutron fluxes also indicates that the three nuclear data libraries have no problem for iron as shown in Figs. 10 and 11, though JENDL-HE/2007 is slightly better than FENDL-3.1b and JENDL-4.0 for the peak neutron fluxes, while JENDL-4.0 is slightly better than FENDL-3.1b and JENDL-HE/2007 for the continuum neutron fluxes.

\subsection{Iron experiment with $65 \mathrm{MeV}$ neutrons}

The measured and calculated neutron spectra in the iron experiment with $65 \mathrm{MeV}$ neutrons are shown in Fig. 12. In this logarithmic scale the calculated neutron spectra seem to agree with the measured ones. However from the quantitative comparison between the measured and calculated neutron fluxes in Figs. 13 and 14, it is noted that the $\mathrm{C} / \mathrm{E}$ tendencies are clearly different among the three nuclear data libraries : the calculated neutron fluxes with FENDL-3.1b agree with the measured ones the best, while those with JENDL-4.0/HE are smaller by $\sim 40 \%$ and $\sim 20 \%$ at maximum than those with FENDL-3.1b and JENDL-HE/2007, respectively.

\subsection{Problems of ${ }^{56} \mathrm{Fe}$ data in JENDL-4.0/HE}

In order to specify reasons for the larger underestimation of the calculation result with JENDL-4.0 in the iron experiment with $65 \mathrm{MeV}$ neutrons, the cross section data of ${ }^{56} \mathrm{Fe}$, which is a main iron isotope, above $20 \mathrm{MeV}$ in FENDL-3.1b, JENDL/HE-2007 and JENDL-4.0/HE are compared. Note that the cross section data of ${ }^{56} \mathrm{Fe}$ above $20 \mathrm{MeV}$ are only non-elastic scattering cross sections and elastic scattering cross sections as shown in Figs. 15 and 16. The non-elastic scattering cross section data in JENDL-4.0/HE are larger than those in FENDL-3.1b and JENDL/HE-2007, while the elastic scattering cross section data in JENDL-4.0/HE are smaller than those in FENDL-3.1b and JENDL/HE-2007.

Thus we temporarily produce two nuclear data, JENDL-4.0/HE non-elas and JENDL-4.0/HE elas, where non-elastic scattering and elastic scattering data above 20 $\mathrm{MeV}$ of the ${ }^{56} \mathrm{Fe}$ data in JENDL-4.0/HE are replaced with those in FENDL-3.1b, respectively, and process them 


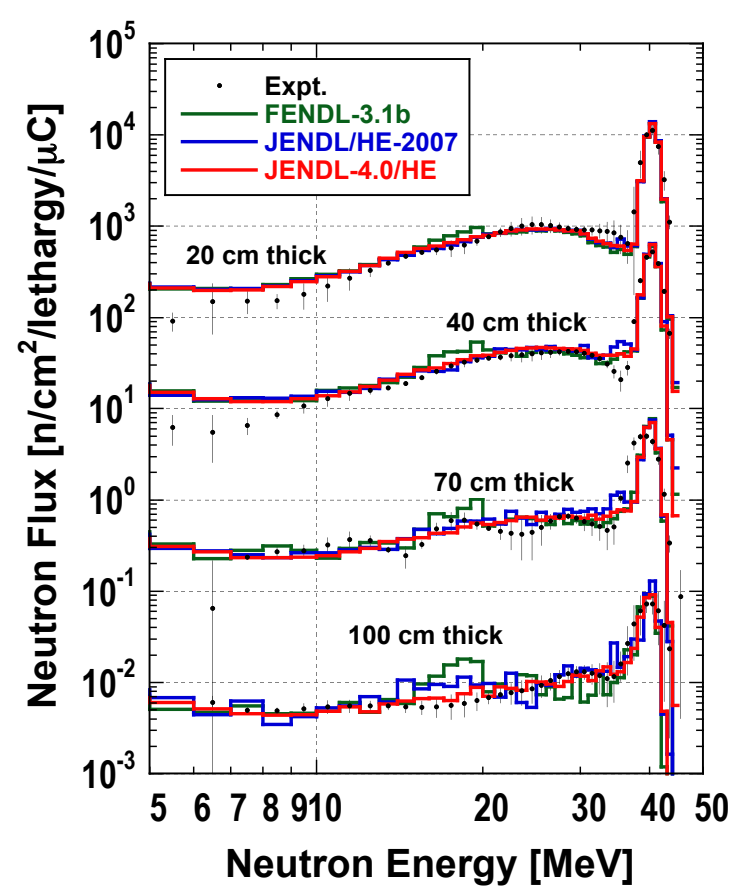

Fig. 9. Measured and calculated neutron spectra in iron experiment with $40 \mathrm{MeV}$ neutrons.

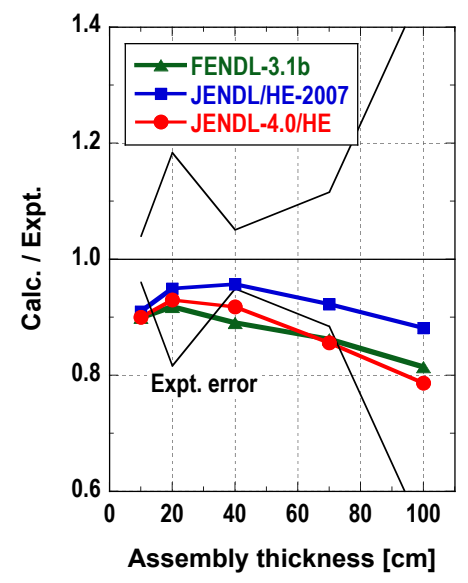

Fig. 10. C/Es of peak (35-45 MeV) neutron fluxes in iron experiment with $40 \mathrm{MeV}$ neutrons.

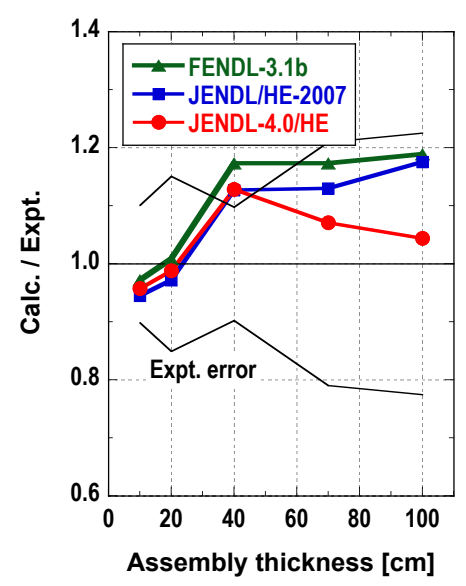

Fig. 11. C/Es of continuum (10-35 MeV) neutron fluxes in iron experiment with $40 \mathrm{MeV}$ neutrons.

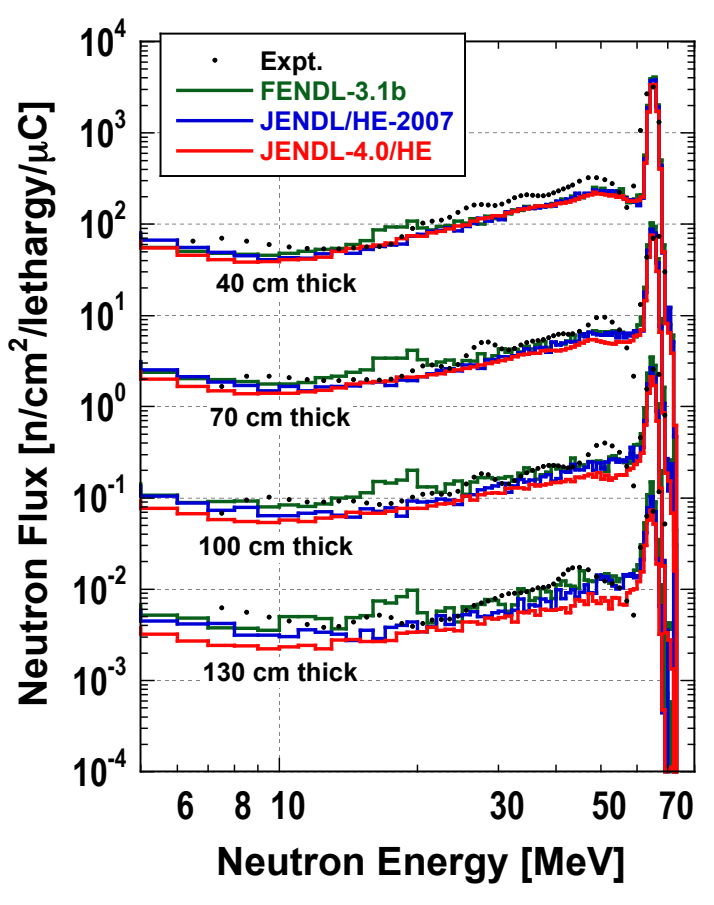

Fig. 12. Measured and calculated neutron spectra in iron experiment with $65 \mathrm{MeV}$ neutrons.

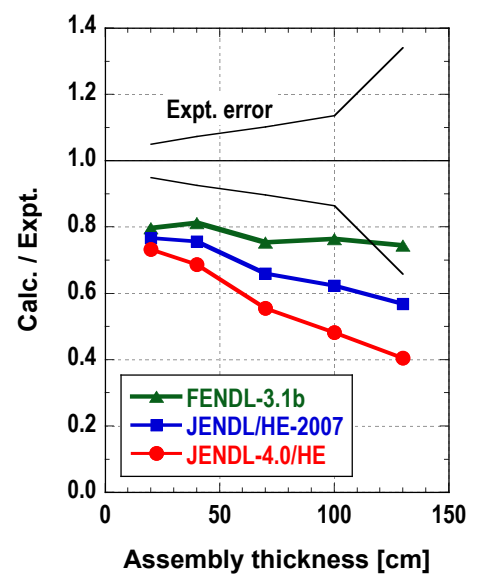

Fig. 13. C/Es of peak (60-70 MeV) neutron fluxes in iron experiment with $65 \mathrm{MeV}$ neutrons.

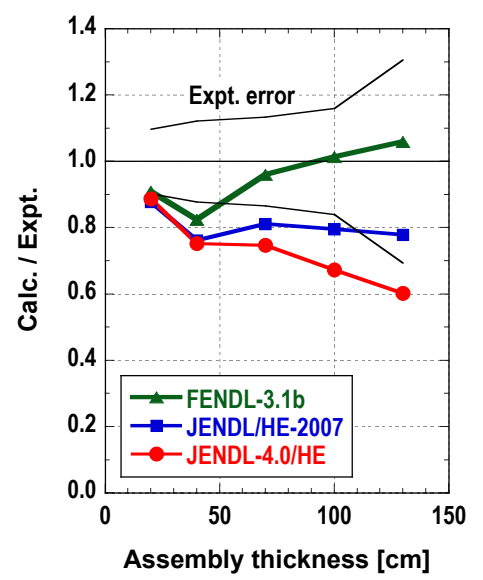

Fig. 14. C/Es of continuum (10-60 MeV) neutron fluxes in iron experiment with $65 \mathrm{MeV}$ neutrons. 


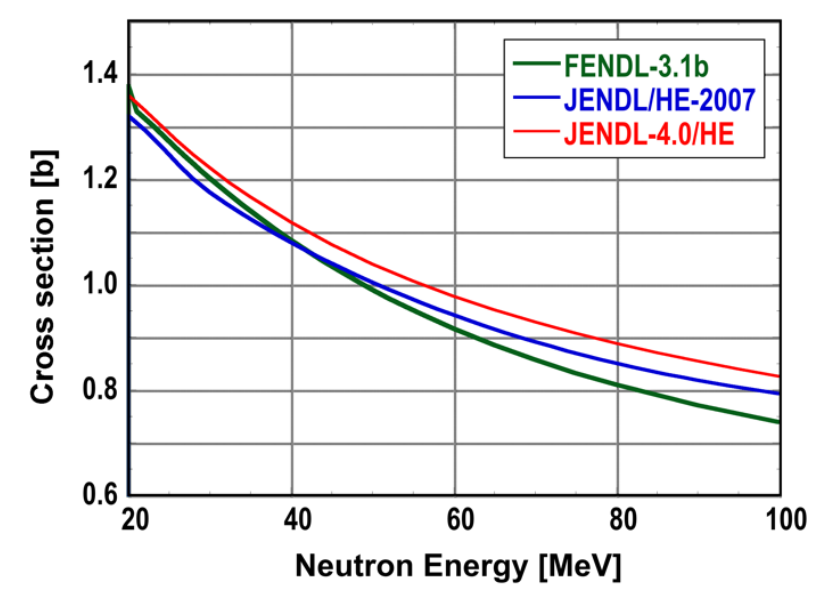

Fig. 15. Non-elastic scattering cross sections of ${ }^{56} \mathrm{Fe}$.

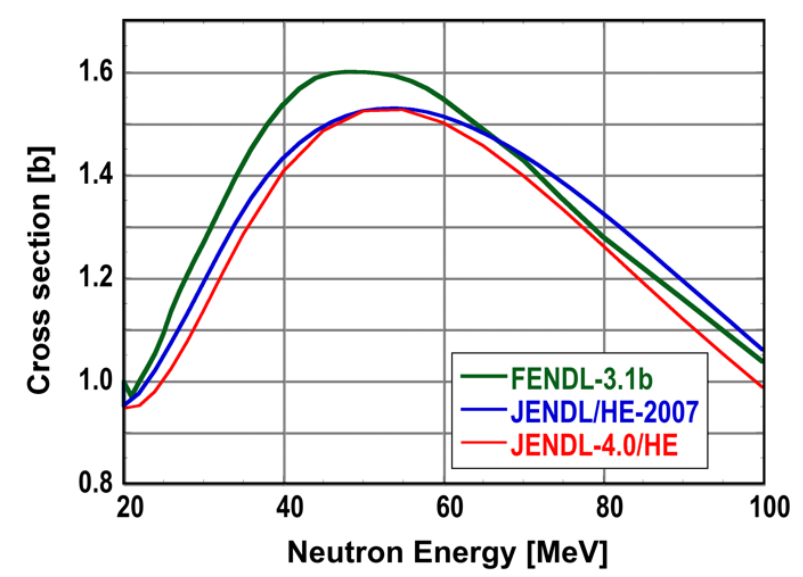

Fig. 16. Elastic scattering cross sections of ${ }^{56} \mathrm{Fe}$.

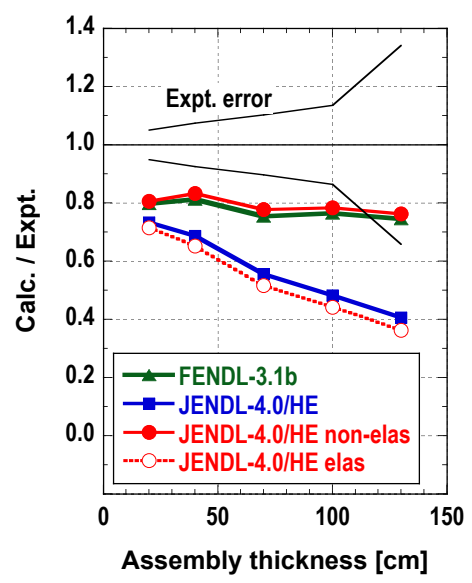

Fig. 17. C/Es of peak (60-70 MeV) neutron fluxes with JENDL-4.0/HE non-elas and JENDL-4.0/HE elas in iron experiment with $65 \mathrm{MeV}$ neutrons.

with NJOY2012.50. The calculation results with JENDL4.0/HE non-elas and JENDL-4.0/HE elas for the iron experiment with $65 \mathrm{MeV}$ neutrons are shown in Figs. 17 and 18. The calculation result with JENDL-4.0/HE nonelas is almost the same as that with FENDL-3.1b. On the other hand, the calculation result with JENDL-4.0/HE elas is almost the same as that with JENDL-4.0/HE. It is

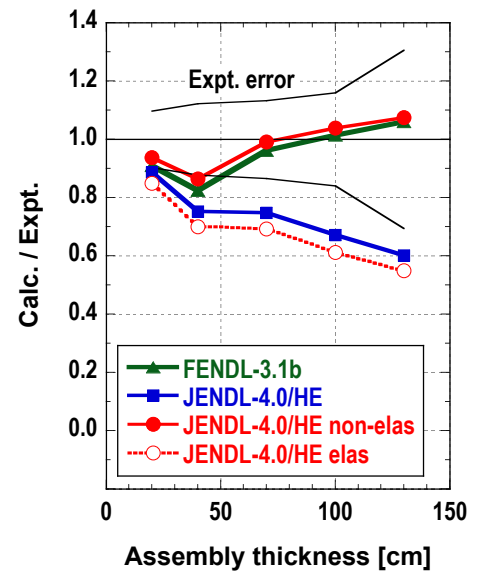

Fig. 18. C/Es of continuum (10-60 MeV) neutron fluxes with JENDL-4.0/HE non-elas and JENDL-4.0/HE elas in iron experiment with $65 \mathrm{MeV}$ neutrons.

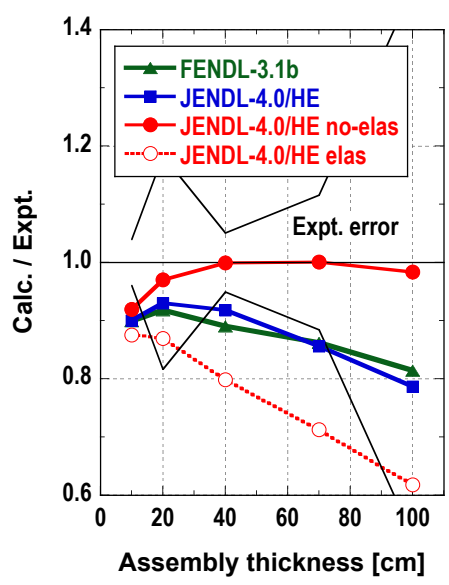

Fig. 19. C/Es of peak (35-45 MeV) neutron fluxes with JENDL-4.0/HE non-elas and JENDL-4.0/HE elas in iron experiment with $40 \mathrm{MeV}$ neutrons.

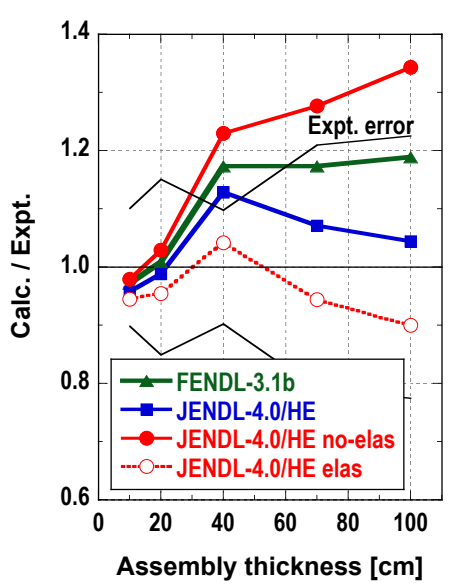

Fig. 20. C/Es of continuum (10-35 MeV) neutron fluxes with JENDL-4.0/HE non-elas and JENDL-4.0/HE elas in iron experiment with $40 \mathrm{MeV}$ neutrons.

considered that the smaller neutron fluxes with JENDL4.0/HE in the iron experiment with $65 \mathrm{MeV}$ neutrons are due to the larger non-elastic scattering cross sections of 
${ }^{56} \mathrm{Fe}$ around $65 \mathrm{MeV}$.

However the results for the iron experiment with 40 MeV neutrons are different as shown in Figs. 19 and 20. The calculation result with JENDL-4.0/HE non-elas is larger than those with FENDL-3.1b and JENDL-4.0/HE and that with JENDL-4.0/HE elas is smaller than those with FENDL-3.1b and JENDL-4.0/HE. It is considered that the calculation result with JENDL-4.0 agrees with that with FENDL-3.1b as a result of accidental cancellation of the overestimation and underestimation.

\section{Conclusions}

We analyzed the concrete and iron shielding experiments at JAEA/TIARA with JENDL-4.0/HE, JENDL/HE-2007 and FENDL-3.1b for benchmarking JENDL-4.0/HE. The calculation result with JENDL-4.0/HE agreed with the measured one for the concrete experiments. It also agreed with the measured one for the iron experiment with 40 $\mathrm{MeV}$ neutrons, but it underestimated the measured neutron fluxes in the iron experiment with $65 \mathrm{MeV}$ neutrons. The comparison of the ${ }^{56} \mathrm{Fe}$ data among JENDL4.0/HE, JENDL/HE-2007 and FENDL-3.1b indicated that the non-elastic scattering cross section data in JENDL-4.0/HE were larger than those in JENDL/HE2007 and FENDL-3.1b around $65 \mathrm{MeV}$, which caused the underestimation of neutron fluxes in the iron experiment with $65 \mathrm{MeV}$ neutrons. The ${ }^{56} \mathrm{Fe}$ data in JENDL-4.0/HE should be revised based on this study.

\section{References}

1. S. Kunieda et al., Japan Atomic Energy Agency report, JAEA-Conf 2008-004, 41 (2016)

2. Y. Nakane et al., Japan Atomic Energy Research Institute report, JAERI-Data/Code 96-029 (1996)

3. K. Kondo et al., Prog. Nucl. Sci. Technol. 4, 322 (2014)

4. R.A. Forrest et al., International Nuclear Data Committee report, INDC(NDS)-0628 (2012)

5. Y. Watanabe et al., J. Korean Phys. Soc. 59, 1040 (2011)

6. M.B. Chadwick et al., Nuclear Data Sheets 112, 2887 (2011)

7. C. Konno, et al., Fusion Eng. Design 98-99, 2178 (2015)

8. X-5 Monte Carlo Team, Los Alamos National Laboratory report, LA-UR-03-1987 (2005)

9. A.C. Kahler et al., Los Alamos National Laboratory report, LA-UR-12-27079 (2012)

10. T. Sasa et al., Japan Atomic Energy Agency report, JAEA-Data/Code 2008-022 (2008)

11. https://www-nds.iaea.org/fendl/ 